\title{
Review
}

\section{Terahertz time} domain spectroscopy and imaging: Emerging techniques for food process monitoring and quality control

\section{A.A. Gowen ${ }^{\mathrm{a}, \mathrm{b}, *}$, C. O'Sullivan ${ }^{\mathrm{c}}$ and C.P. O'Donnell ${ }^{a}$}

\author{
a University College Dublin, School of Biosystems \\ Engineering, Earlsfort Terrace, Dublin, Ireland

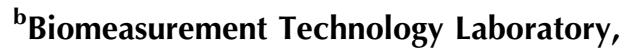 \\ Kobe University, Japan \\ (Tel.: + 3531 7167413; fax: + 3531 7167415; \\ e-mail: aoife.gowen@ucd.ie) \\ cDept. of Experimental Physics, NUI Maynooth, \\ Maynooth, Co. Kildare, Ireland
}

The Terahertz ( $\mathrm{THz}$ ) region of the electromagnetic spectrum, spanning the range between $100 \mathrm{GHz}$ and $30 \mathrm{THz}$, has recently enjoyed a renaissance due to technological developments in source and detector components. With the development of $\mathrm{THz}$ instrumentation, applications of $\mathrm{THz}$ spectroscopy and imaging for quality control of food products have expanded in scope and improved in performance. This article gives an overview of the fundamentals of $\mathrm{THz}$ technology and a comprehensive review of applications of $\mathrm{THz}$ time domain spectroscopy and imaging for food quality and control. Technical challenges and future outlook for these emerging techniques are also discussed.

\section{* Corresponding author.}

0924-2244/\$ - see front matter @ 2012 Elsevier Ltd. All rights reserved. doi:10.1016/j.tifs.2011.12.006

\section{Introduction}

Once known as the Terahertz (THz) gap, the region of the electromagnetic spectrum from $100 \mathrm{GHz}$ to $30 \mathrm{THz}$ was largely ignored until the mid-1990s. This was due to the low efficiency of sources and detectors of $\mathrm{THz}$ energy which made it difficult to build efficient instrumentation in this wavelength range. The development of ultrafast lasers in the 1990s was a major turning point, leading to the development of modern day THz-time domain spectroscopy (Thz-TDS). THz systems can now be developed using a variety of sources, such as Gunn diodes (Chandra et al., 1997) and super-lattice electronic devices (Renk et al., 2007), which operate at the low frequency end of the $\mathrm{THz}$ band, or photonic sources, such as lasers and photoconductive dipole antennae (Tani et al., 2002), which are more widespread, and can generate radiation across a broadband frequency range. Other $\mathrm{THz}$ generation methods include optical rectification (Radhanpura et al., 2009) and mixing of light from two laser sources whose wavelength difference is in the $\mathrm{THz}$ region.

Terahertz systems can be designed to operate in transmission or reflection mode and in the time or spatial domain. Terahertz radiation has sufficient energy $(1-10 \mathrm{meV})$ to promote rotations and vibrations of molecules and can thus be used for their identification; for example, Thz-TDS has been related to the function of biomolecules and conformations of proteins (Kim, Born et al., 2008; Kim, Jeon et al., 2008; Markels et al., 2002). It is non-ionising and sensitive to polar molecules such as water. At the same time, THz radiation interacts only very weakly with materials composed of non-polar molecules, such as plastics and ceramics and is reflected by most metals. This means that THz can 'see' through common packaging materials and textiles, making it well suited for security operations such as passenger screening at airport terminals. Many biological molecules have distinct $\mathrm{THz}$ signatures. He et al. (2006) demonstrated the potential of Thz-TDS in the range $0.1-2.5 \mathrm{THz}$ for characterisation of animal tissue. Based on power absorption coefficients extracted from Thz-TDS spectra, they showed that skin, fat and lean tissues of the same animal exhibited different frequency dependent response, due to variations in water content, while similar tissues from different animals (rats and pigs in this case) showed similar responses. McAuley et al. (2006) demonstrated THz transmission imaging of bacon slices, and clear distinction between fatty and lean tissues was possible. 
Spectroscopic methods such as NIR spectroscopy and chemical imaging are of major interest to industries such as pharmaceutical and food (Gowen et al., 2007, 2008; Shen, 2011), as they facilitate rapid, non-destructive monitoring of inter and intra batch variability in processing methods. This is of major importance in quality control and allows manufacturers to benefit from the adoption of the process analytical technology (PAT) paradigm. The US Food and Drug Administration-led PAT initiative is transforming approaches to quality assurance in industry. Core to the PAT initiative is increased process understanding by monitoring of critical performance attributes, leading to better process control and ultimately improved product quality. Potential advantages of PAT implementation include: reduced production cycle times, prevention of rejects, reduction of human error and facilitation of continuous processing to improve efficiency. Compared with other vibrational spectroscopic methods, such as NIR and MIR spectroscopy, Teraherz spectroscopy has the distinct advantage that it can penetrate many organic materials (e.g. plastics, paper, skin). Commercially available Fourier transform infrared (FTIR) spectrometers can operate in the spectral range down to $1.5 \mathrm{Thz}$. However, the dynamic range of far infrared FTIR spectroscopy is limited due to source and detector efficiency. Unlike NIR chemical imaging, $\mathrm{THz}$ imaging enables direct measurement of sample thickness, refractive index and absorption coefficient. However, at the present time, commercial NIR imaging systems are at a more advanced state of development than $\mathrm{THz}$ systems. Examples of $\mathrm{THz}$ imaging applied to pharmaceutical quality control include tablet coating characterisation, evaluation of dissolution properties and characterisation of polymorphisms (Shen, 2011).
The number of reported applications of $\mathrm{THz}$ for food industry-related research has increased in tandem with those in the pharmaceutical industry. It therefore seems timely to provide a review of $\mathrm{THz}$ spectroscopy and imaging applications for food quality monitoring. This article is structured as follows: the next section gives an overview of instrumentation common in $\mathrm{THz}$ spectroscopy; this is followed by a short description of data analysis in THz. The main body of the article is found in Section 4, which compiles the reported applications of $\mathrm{THz}$ spectroscopy and imaging to food quality analysis (a summary is provided in Table 1). This is followed by a discussion of the challenges and limitations of this technology. Some brief conclusions end the article.

\section{Instrumentation}

Terahertz spectroscopy systems can be broadly divided into two categories based on their principle of operation: pulsed and continuous wave systems. A comparison of the advantages and disadvantages of each kind of system is given in Table 2. Pulsed systems rely on the generation of $\mathrm{THz}$ waves by ultrafast lasers which emit sub $100 \mathrm{fs}$ pulses, focussed on photoconductive antennae (PCAs). There are typically two PCAs in a THz system: one emitter and one detector. An external electric field is applied to the emitter PCA in order to maintain an electrical bias. When excited by the fs pulse, the electrically biased emitter PCA emits a THz pulse (Fig. 1). The THz wave generated is typically re-focussed prior to passing through a sample using polymeric lenses or mirrors. After passing through a sample, the beam may be realigned, again by use of polymeric lenses or mirrors, and is directed to the detector PCA. Another portion of the optical pulse is used to gate the

\begin{tabular}{|c|c|c|c|c|}
\hline Product & Author, year & Application & THz modality & THz range \\
\hline Wheat & Chua et al., 2004, 2005 & Moisture content & TDS & $0.1-4$ \\
\hline Wafers & Parasoglou et al., 2009 & Moisture content & TDS & $0.1-2.4$ \\
\hline $\begin{array}{l}\text { Food powders } \\
\text { (sticky rice, } \\
\text { sweet potato, } \\
\text { and lotus root) }\end{array}$ & Hua \& Zhang, 2010 & Pesticide detection & TDS & $0.5-1.6$ \\
\hline Animal feed & (Redo-Sanchez et al., 2011) & Antibiotic detection & TDS & $0.1-2$ \\
\hline Milk powder & (Redo-Sanchez et al., 2011) & Antibiotic detection & TDS & $0.1-2$ \\
\hline Egg powder & (Redo-Sanchez et al., 2011) & Antibiotic detection & TDS & $0.1-2$ \\
\hline Chocolate & (Jordens \& Koch, 2008) & Foreign body detection & Imaging & $0.1-3$ \\
\hline $\begin{array}{l}\text { Vegetable oil } \\
\text { (sunflower, peanut, } \\
\text { soybean and } \\
\text { rapeseed oil) }\end{array}$ & Li (2010) & $\begin{array}{l}\text { Characterization of optical } \\
\text { properties }\end{array}$ & TDS & $0.2-1.5$ \\
\hline $\begin{array}{l}\text { Beverages } \\
\text { (alcohol-free beer, } \\
\text { Danish Pilsner, } \\
\text { Danish Porter beer, } \\
\text { French white wine, } \\
\text { Irish cream liquor, } \\
\text { German Bitter and } \\
\text { Bulgarian Absinthe) }\end{array}$ & Jepsen et al. (2007) & $\begin{array}{l}\text { Prediction of sugar and } \\
\text { alcoholic content }\end{array}$ & TDS & $0.1-1$ \\
\hline
\end{tabular}




\begin{tabular}{|lll|}
\hline \multicolumn{2}{|l|}{ Table 2. Comparison of pulsed and continuous wave THz systems. } \\
\hline & Pulsed & Continuous \\
\hline $\begin{array}{l}\text { Direct measurement of transient } \\
\text { electric field }\end{array}$ & $\mathrm{X}$ & \\
Ambient noise & Low & High \\
Phase information retained & $\mathrm{X}$ & $\mathrm{X}$ \\
$\begin{array}{l}\text { Monitoring of highly dynamic } \\
\text { responses }\end{array}$ & $\mathrm{X}$ & \\
spectral resolution & Low & High \\
Intensity data & & $\mathrm{X}$ \\
Frequency, time, depth data & $\mathrm{X}$ & \\
Speed & High & High \\
Cost & High & Lower \\
Data complexity & High & Low \\
\hline
\end{tabular}

detector PCA. The THz spectrum is generated by delaying the laser pulses going to the emitter and detector. This results in a time delay signal corresponding to one point in the $\mathrm{THz}$ spectrum. By changing the delay and measuring the signal corresponding to each delay the entire waveform is measured. The resultant signal is a measure of both the phase and amplitude of the $\mathrm{THz}$ field. Fourier transformation is used to convert the time-domain signal into the frequency domain. The absorption coefficient and refractive index of the material studied are directly related to the amplitude and phase of the transmitted field. This type of system may be used in transmission or reflection set-up, as shown in Fig. 1(a) and (b). Since Thz-TDS is a phase sensitive technique, in reflection or transmission mode, determination of the complex reflection coefficient can provide information on the dielectric constant of a sample. Recent developments in optical instrumentation have improved the speed of pulsed systems (Jin et al., 2007; Kim et al., 2010; Kim et al., 2008).
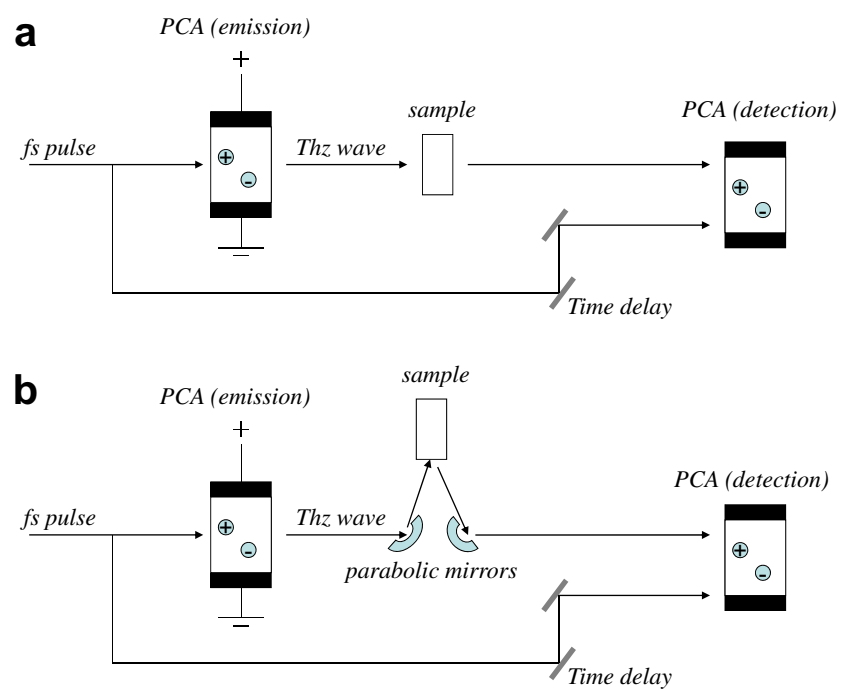

Fig. 1. Schematic showing pulsed $\mathrm{THz}$ acquisition in (a) transmission and (b) Reflection modes.
A typical Thz spectrum is shown in Fig. 2. In order to obtain a THz spectrum of a sample it is necessary to record a reference spectrum. In the case of transmission geometry, the reference is usually obtained by passing light through the system with no sample. For the reflection set-up, the reference is usually obtained by replacing the sample with a material of known reflectance, e.g. a mirror. Alternatively, it is possible to encase the sample in a container and measure the reflection from the air/container and container/ sample interfaces. This method, known as self referencing, removes the need for a separate reference measurement (Jepsen et al., 2007; Redo-Sanchez and Zhang, 2011).

Continuous wave systems do not require fs lasers, since the $\mathrm{THz}$ wave is generated by, for example, combining two frequency stabilised lasers, whose difference is in the $\mathrm{THz}$ region. Such systems are normally less complex and tend to be less expensive. than the pulsed systems, and are useful for detecting sharp spectral features, with resolution typically of a few $\mathrm{MHz}$. $\mathrm{THz}$ imaging systems have also been developed, based on CW and pulsed systems described above. These are typically based on rastering, i.e. moving the sample along the $\mathrm{x}-\mathrm{y}$ dimensions so that the $\mathrm{THz}$ beam passes through different spatial positions on the sample. An image is built up by recording a THz signal for each spatial position. This means that image acquisition is time consuming, varying from a few minutes to hours, depending on system characteristics and the desired spatial resolution. Due to diffraction, the image resolution obtainable is limited by the wavelength of the THz beam applied; however, various near-field set-ups have been proposed to reduce the pixel size to the order of $100 \mathrm{~s}$ of microns (Wang et al., 2009; Yan et al., 2009).

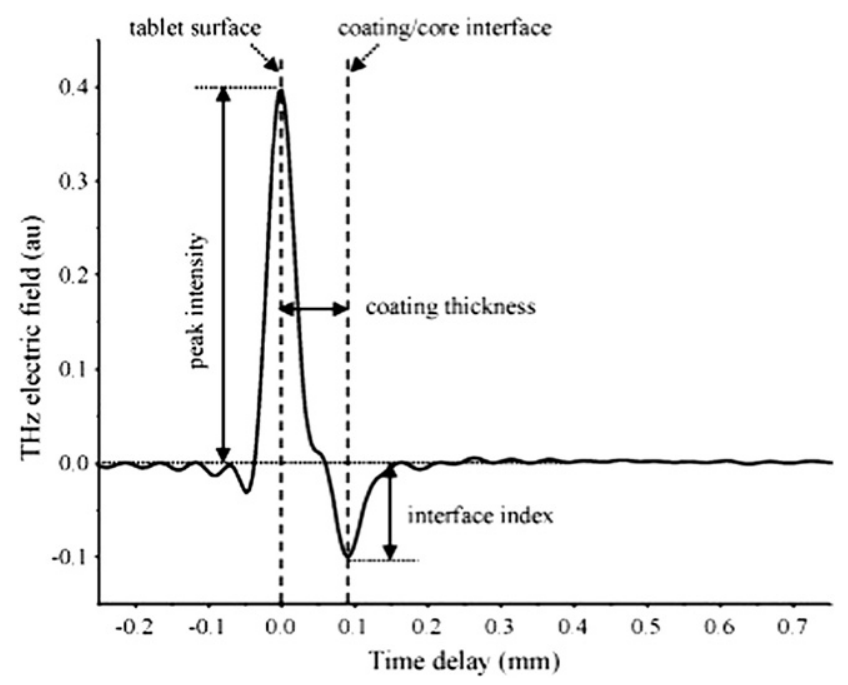

Fig. 2. Typical terahertz waveform. Reflection from the tablet surface (i.e. air/coating interface) and from the coating/core interface are indicated by dashed lines; drawn through arrows indicate how TPI parameters (peak intensity, interface index, layer thickness) are related to the waveform. (Maurer \& Leuenberger, 2009). 


\section{Data analysis}

Once the THz waveform has been acquired and transformed by Fourier transform into the frequency domain, it is necessary to calculate the absorption coefficient and refractive index for the sample under consideration. In the transmission set up, this is achieved using the sample $\left(\mathrm{E}_{\mathrm{s}}(\mathrm{v})\right)$ and reference $\left(\mathrm{E}_{\mathrm{r}}(\mathrm{v})\right)$ spectra. The transmission function in TDS is defined as follows (Hua \& Zhang, 2010):

$$
\begin{aligned}
\mathrm{T}(\mathrm{v}) & =\mathrm{E}_{\mathrm{s}}(\mathrm{v}) / \mathrm{E}_{\mathrm{r}}(\mathrm{v}) \\
& =\left[4 \mathrm{n} /(\mathrm{n}+1)^{2}\right] \exp [-\alpha \mathrm{d} / 2+\mathrm{i} 2 \pi \mathrm{v}(\mathrm{n}-1) \mathrm{d} / \mathrm{c}] \\
& =\operatorname{Aexp}(\mathrm{i} \Phi(\mathrm{v}))
\end{aligned}
$$

where $\mathrm{d}$ is the sample thickness, $\mathrm{v}$ is the radiation frequency, $\mathrm{c}$ is the speed of light in a vacuum, $\mathrm{n}$ is the refractive index, $\alpha$ is the absorption coefficient, $A$ is the amplitude ratio between the sample and reference spectra and $\phi$ is the relative phase difference. Equating the real and imaginary portions of Eq. (1), it is possible to calculate $\mathrm{n}$ and $\alpha$ for each frequency as follows:

$\mathrm{n}=1+\mathrm{c} \Phi /(2 \pi \mathrm{vd})$

$a=(2 / d) \ln \left[4 n /(n+1)^{2} / A\right]$

Certain compounds, such as pesticides or antibiotics, may exhibit sharp absorption peaks at specific $\mathrm{THz}$ frequencies. These features can be used for detection of contaminants in foodstuffs, and several examples are presented in the next section. Chemometric analysis (e.g. partial least squares regression) of the absorption coefficient or refractive index is also often applied for quantitative analysis of samples (Hua \& Zhang, 2010; Nakajima et al., 2007; Otsuka et al., 2010; Shen, 2011).

\section{Applications in food quality control}

Moisture content determination

Due to the high absorbance of $\mathrm{THz}$ radiation by water, the most obvious application of $\mathrm{THz}$ spectroscopy would seem to be for quantification of moisture content in foods. This is especially important in controlling drying processes where low moisture content can alter the sensory qualities and shelf life of products. Moreover, the possibility of characterising the molecular nature of water in low moisture content products (e.g. free versus bound water) could be used for more accurate determination of shelf life.

Chua et al. (2004) and Chua et al. (2005) investigated the application of Thz-TDS in the $0.1-4 \mathrm{THz}$ range for evaluation of moisture content of whole and crushed wheat. The crushed samples were housed in a PTFE sample holder during measurement, since this polymer is transparent to $\mathrm{THz}$ radiation, and the system was purged with nitrogen gas to avoid absorption by water vapour. Scattering of $\mathrm{THz}$ light due to the shape and orientation of grains led to the appearance of apparent peaks in the absorbance spectrum. Such artefacts were not present in the spectra of crushed wheat, and it was possible to identify waterspecific absorption peaks in crushed samples varying in moisture content from 12 to $18 \%$. Subtracting the $\mathrm{THz}$ spectrum of dry crushed wheat from that of wetted samples facilitated the development of a calibration model for prediction of moisture.

The moisture content of food wafers has also been examined using Thz-TDS (Parasoglou et al., 2009). Dehydrated wafers were brought to various moisture contents $(1-30 \%)$ by storage in a humid environment. Samples were then encased in sealed cells during for THz transmission measurement, in order to avoid dehydration by nitrogen purging of the $\mathrm{THz}$ system. A linear relationship between moisture content and $\mathrm{THz}$ time domain data (peak to peak amplitude) or frequency domain data in the range $0.2-0.6 \mathrm{THz}$ was reported.

Although the potential for Thz-TDS has been demonstrated for several low moisture content foods, there have been few, if any, reported applications of $\mathrm{THz}$ imaging for moisture detection. Such investigations would be valuable for determining the spatial distribution of moisture in a food, which could be further related to processing conditions. A related application has been reported: $\mathrm{THz}$ imaging for detection of moisture content of paper (Banergee et al., 2008). In this work, a raster based single pixel detection system was employed to map the distribution of moisture in paper at a spatial resolution in the mm range. An image representing $40 \times 40 \mathrm{~mm}$ of paper was obtained within $2 \mathrm{~min}$. Although the imaging of larger sheets of paper using this method would be prohibitively time consuming, these results suggest the feasibility of obtaining similar $\mathrm{THz}$ images for smaller, thin, low moisture content foods such as wafers, pasta or pastry sheets.

\section{Residue detection}

The presence of residues in foods is becoming a growing concern among consumers. Consequently, there has been interest in developing rapid non-destructive techniques for residue detection in foods, and $\mathrm{THz}$ spectroscopy is being evaluated for this purpose. Hua and Zhang (2010) examined Thz-TDS as a non-invasive tool for the detection of pesticides in food powders (sticky rice, sweet potato, and lotus root)(Hua and Zhang). They demonstrated that four pesticides (imidacloprid, carbendazim, tricylazole, and buprofezin) had specific absorbance peaks in the $\mathrm{THz}$ range $(0.5-1.6 \mathrm{THz})$, while the spectra of the food powders themselves were similar to each other, exhibiting broad features. This indicated that the pesticide samples could be easily distinguished both from each other and from the food powder matrices. For instance, a linear correlation between absorbance at $0.89 \mathrm{THz}$ and imidacloprid in sticky rice powder was reported. Partial least squares regression was carried out to predict imidacloprid concentration from the full spectrum, leading to promising results $\left(R^{2}>0.99\right)$. However, it was noted that the physical properties of food 
matrix (sticky rice powder in this case) can alter the signal due to scattering and absorption. In order to verify this method, the measurement of a wider range of physical conditions and food matrices is required.

More recently, Redo-Sanchez et al., 2011 investigated the potential of Thz-TDS for the detection of antibiotic residues in foodstuffs. Standard mixtures of antibiotics and powdered polyethelyne (transparent to $\mathrm{THz}$ energy) were prepared in pelletized form and transmission Thz-TDS spectra were obtained in the range 0.1 and 2 THz. Eight of the eleven antibiotics tested had specific signatures in the $\mathrm{THz}$ region, while two of them (doxycycline and sulfapyridine) were detectable when mixed with different food matrices (animal feed, milk, and egg powder). This indicates the potential for detection of some antibiotics in food substances using Thz-TDS. However, the mixture ratios used in this study were high (containing minimum $50 \%$ antibiotic content); further research is required to determine limits of detection for this application.

\section{Foreign body detection}

Detection of metallic contamination is rather straightforward in food processing; however, detection of non- metallic contamination, such as glass or plastic, is more challenging. The implementation of imaging systems with high spatial resolution would allow identification of the precise location of foreign bodies, thus minimising rejects. However, existing methods such as X-ray imaging do not perform well when the densities of the foreign body and the food product are similar. $\mathrm{THz}$ imaging, providing both phase and amplitude information, offers improved characterisation of foreign bodies. One demonstration of $\mathrm{THz}$ imaging for foreign body detection in foods has been reported, for the detection of contaminants in chocolate (Jordens \& Koch, 2008). Due to its high fat and low moisture content, chocolate is relatively transparent to $\mathrm{THz}$ energy. When foreign objects, such as glass or plastic, are placed in chocolate, they alter the scattering profile of a transmitted $\mathrm{THz}$ wave and are thus detectable. This observation formed the basis of the work of Jordens \& Koch, 2008. Foreign bodies (glass, stone and metal) were concealed within the interior of a bar of chocolate and $\mathrm{THz}$ images of the contaminated sample were obtained using a raster scanning Thz-TDS system operating in transmission mode. It was possible to identify foreign bodies in the chocolate sample, both in the presence and absence of its plastic foil packaging. However, variations in the chocolate thickness, caused by the morphology of the chocolate bar, resulted in variations in the transmitted signal. Another limitation highlighted in this study was the speed of $\mathrm{THz}$ image acquisition. The authors describe a method that could be implemented in order to speed up the process of imaging by sampling points rather than acquiring the entire waveform. In order to overcome the effect of sample thickness on signal variation, the authors suggest using two time delays. It was reported that such a method could facilitate scanning speeds up to $0.55 \mathrm{~m} / \mathrm{s}$.

\section{Oil characterisation}

Detection and characterization of water contamination of oils and other products is possible with $\mathrm{THz}$ spectroscopy. Gorenflo et al. (2006) employed Thz-TDS in transmission mode for characterization of the dielectric properties of oil-water complexes with water content ranging from 0.43 to $3.28 \%$. This enabled determination of the absorption coefficient and refractive index of the samples, and thus determination of the amount and structure of water in these complexes. The results indicated the formation of a hydrogen bonded oil-water complex, rather than dissolved water clusters in the oil-water complexes. This represents an important advance in the understanding of the behaviour of water in oil, made possible by $\mathrm{THz}$ spectroscopy. More recently, Cunnell et al. (2009) developed a THz system capable of online measurements for water characterization in oil emulsions. This system, operating in transmission mode, included a quantum cascade laser source operating at $2.7 \mathrm{THz}$ and a specially designed flow through cell for online measurements. It was reported that this system is capable of measuring variations in moisture content of approximately $0.1 \%$ in oil emulsions containing less than $3 \%$ water.

$\mathrm{Li}$ (2010) measured the optical properties of four types of vegetable oil using Thz-TDS in the range 0.2-1.5 THz. The oil samples (sunflower, peanut, soybean and rapeseed oil) were placed in a quartz cuvette for transmission measurement and the empty cuvette was used for reference measurement in this case. Due to multiple reflections at the air-cuvette and oil cuvette interfaces, it was necessary to develop a novel iterative algorithm for extraction of absorption coefficient and refractive index.

\section{Beverage characterisation}

It is important to characterise the dielectric properties of liquid foods in order to optimise certain processes, such as microwave heating. In addition, dielectric properties can be related to compositional information. Jepsen et al. (2007) developed a Thz-TDS system, operating in reflection in the $0.1-1.0 \mathrm{THz}$ frequency range for measurement of the dielectric properties of liquids, in particular aqueous sugar and alcohol solutions. The developed system was employed for simultaneous determination of the sugar and alcohol content of commercial alcoholic beverages independent of other properties such as colour, organic matter content, carbonation and flavour.

\section{Inspection of packed goods}

Common packaging materials made from cardboard and polymers are transparent to $\mathrm{THz}$ radiation. This makes $\mathrm{THz}$ spectroscopy and imaging attractive tools for quality validation of packaged products. Although it is possible to apply some hyperspectral imaging techniques to packaged 
goods, such analysis is typically limited to thin layer polymer packaging and imaging in the visible range (Gowen, O'Donnell, et al., 2010; Gowen, Tiwari, et al., 2010). As demonstrated by Jordens \& Koch, 2008, it is possible to detect foreign bodies in packaged chocolate bars. Indeed, for most if not all of the applications listed in the preceding sections, it is possible to carry out the measurement while a product is cased in glass, plastic or paper. Another potential area of application is in the detection of production defects in packaging (e.g. holes or tears)(Morita et al. 2005).

\section{Challenges and limitations}

A major barrier to the adoption of Terahertz imaging systems at present is the high cost of detectors and sources. Although new developments have drastically decreased these costs, they are currently too high to render the technology economically beneficial for many applications. Related to this is the low acquisition speed typical of most systems. Although it has been demonstrated by Jordens \& Koch, 2008 that high speed systems are feasible, many of these are based on sampling a sub-region of the $\mathrm{THz}$ waveform. The development of such systems is generally applicationspecific, since the sampled sub-region would need to match the desired response. Relatively high signal to noise ratio is also still problematic for certain regions of the $\mathrm{THz}$ spectrum, although this may be ameliorated by acquisition of multiple scans and averaging (although this increases the acquisition time). A number of technology providers are addressing these issues and developing improved, turnkey THz systems (Mantsch \& Naumann, 2010).

Wu et al. (2007) listed a number of challenges, in the form of questions, to be addressed prior to the adoption of $\mathrm{THz}$ as a process analytical tool for the pharmaceutical industry. These challenges are equally relevant for the adoption of $\mathrm{THz}$ in the food industry, and are paraphrased below:

- How do measurement conditions affect quality, precision, accuracy and repeatability of $\mathrm{THz}$ spectra? For instance, in many cases, the $\mathrm{THz}$ system is encased and flushed with nitrogen gas - this may not be feasible in a food processing scenario.

- How can these measurement conditions be optimized for process monitoring? These will vary according to the process of interest; therefore an extensive research and development phase will be required for each application. This is often seen as a barrier in industry as it is expensive in terms of time and money.

- How can process and product knowledge be implemented into the data analysis to improve understanding of the $\mathrm{THz}$ spectra? Again, this is a process/product specific question and is best answered by collaborative research between the instrument provider and user.

- Can other spectroscopic techniques and chemometric modelling be integrated with $\mathrm{THz}$ for improved understanding of product and process interactions? Fusing complementary spectroscopic techniques to optimize process monitoring capabilities could be regarded as the next major challenge in the implementation of PAT strategies for food quality control.

One of the limitations of $\mathrm{THz}$ spectroscopy for moisture content detection is that it is not suitable for high moisture products of thickness greater than $1 \mathrm{~mm}$. This is due to the high absorption of $\mathrm{THz}$ radiation by water. For example, $\mathrm{He}$ et al. (2006) reported that the $\mathrm{THz}$ spectra of thin slices of skin and lean tissue samples were very weak due to water absorption. Even thin samples (approx $1 \mathrm{~mm}$ in thickness) were approximately opaque to $\mathrm{THz}$ above $0.7 \mathrm{THz}$. Reflection $\mathrm{THz}$ imaging is another option; however, the appearance of standing waves due to differences in the optical path length can produce image artefacts (McAuley et al., 2006). Another challenge facing practitioners of $\mathrm{THz}$ spectroscopy is the effect of physical variations in a sample (e.g. particle size) on the refractive index of a material. This is particularly pertinent in the case of quality monitoring of fresh produce which shows high variability in this respect. Scattering effects may adversely affect measurements of Thz absorption in certain materials. When the grain size of solids is comparable to the $\mathrm{THz}$ wavelength, extinction spectra are severely influenced by scattering losses. Bandyopadhyay et al. (2007) investigated the effects of scattering on Thz-TDS spectra (in the range 0.2-1.2 Thz) of granular solids (Ammonium Nitrate, flour and salt) as a function of grain size (Bandyopadhyay et al., 2007). Mie theory was applied to separate the contributions to the signal arising from absorption and scattering, thus enabling the total extinction spectra for various materials of different grain sizes to be extracted. However, the authors demonstrate that Mie theory cannot be successfully applied in the case of large non-speherical particle sizes.

\section{Conclusions and future outlook}

The ability of $\mathrm{THz}$ waves to pass through a wide variety of packaging materials, combined with their ability to characterise the molecular structure of many biological substances makes it an attractive process analytical tool for enhanced monitoring of food production. Although the potential of Thz-TDS spectroscopy and imaging has been demonstrated for a number of issues in food quality control, it is clear that much remains to be investigated in this area. The development of $\mathrm{THz}$ spectral library databases is something that is still in its infancy. Important work has already commenced on this, as evidenced by the applications described in the previous section. Although plenty more remains to be done, with the increased availability of turnkey $\mathrm{THz}$ systems, it is likely that the establishment of a library for the wide group of compounds related to food safety will be accomplished in the not too distant future. This, combined with lowering costs for sources and detectors, and faster systems, should lead to the increased adoption of $\mathrm{THz}$ spectroscopy and imaging for food process monitoring and control. 


\section{Acknowledgements}

Dr. Aoife A. Gowen acknowledges funding from the EU FP7 under the Marie Curie Outgoing International Fellowship.

\section{References}

Bandyopadhyay, A., Sengupta, A., Barat, R. B., Gary, D. E., \& Federici, J. F. (2007). Effects of scattering on THz spectra of granular solids. International Journal of Infrared and Millimeter Waves, 28(11), 969-978.

Banerjee, D., von Spiegel, W., Thomson, M. D., Schabel, S., \& Roskos, H. G. (2008). Diagnosing water content in paper by terahertz radiation. Optics Express, 16(12), 9060-9066.

Chandra, I., Gulati, R., \& Sharma, H. S. (1997). Advances in Gunn diode technology. lete Technical Review, 14(6), 443-449.

Chua, H. S., Obradovic, J., Haigh, A. D., Upadhya, P. C., Hirsch, O., Crawley, D., et al. (2005). Terahertz time-domain spectroscopy of crushed wheat grain. In: Microwave Symposium Digest, 2005. IEEE MTT-S International.

Chua, H.S., Upadhya, P.C., Haigh, A.D., Obradovic, J., Gibson, A.A.P., \& Linfield, E.H. (2004). Terahertz time-domain spectroscopy of wheat grain. Infrared and Millimeter Waves, 2004 and 12th International Conference on Terahertz Electronics, 2004. Conference Digest of the 2004 Joint 29th International Conference on.

Cunnell, R., Luce, T., Collins, J., Rungsawang, R., Freeman, J.R., Beere, H.E., et al. (2009). Quantification of emulsified water content in oil using a terahertz quantum cascade laser. Infrared, Millimeter, and Terahertz Waves, 2009. IRMMW-THz 2009. 34th International Conference on.

Gorenflo, S., Tauer, U., Hinkov, I., Lambrecht, A., Buchner, R., \& Helm, H. (2006). Dielectric properties of oil-water complexes using terahertz transmission spectroscopy. Chemical Physics Letters, 421(4-6), 494-498.

Gowen, A. A., O'Donnell, C. P., Cullen, P. J., Downey, G., \& Frias, J. M. (2007). Hyperspectral imaging - an emerging process analytical tool for food quality and safety control. Trends in Food Science \& Technology, 18(12), 590-598.

Gowen, A. A., O'Donnell, C. P., Cullen, P. J., \& Bell, S. E. J. (2008). Recent applications of chemical imaging to pharmaceutical process monitoring and quality control. European Journal of Pharmaceutics and Biopharmaceutics, 69(1), 10-22.

Gowen, A. A., O'Donnell, C. P., Esquerre, C., \& Downey, G. (2010). Influence of polymer packaging films on hyperspectral imaging data in the visible-near-infrared $(450-950 \mathrm{~nm})$ wavelength range. Applied Spectroscopy, 64(3), 304-312.

Gowen, A. A., Tiwari, B. K., Cullen, P. J., McDonnell, K., \& O'Donnell, C. P. (2010). Applications of thermal imaging in food quality and safety assessment. Trends in Food Science \& Technology, 21(4), 190-200.

He, M., Azad, A. K., Ye, S., \& Zhang, W. (2006). Far-infrared signature of animal tissues characterized by terahertz time-domain spectroscopy. Optics Communications, 259(1), 389-392.

Hua, Y. F., \& Zhang, H. J. (2010). Qualitative and quantitative detection of pesticides with terahertz time-domain spectroscopy. IEEE Transactions on Microwave Theory and Techniques, 58(7), 2064-2070.

Jepsen, P. U., Møller, U., \& Merbold, H. (2007). Investigation of aqueous alcohol and sugar solutions with reflection terahertz timedomain spectroscopy. Optics Express, 15(22), 14717-14737.

Jin, Y. S., Jeon, S. G., Kim, G. J., Kim, J. I., \& Shon, C. H. (2007). Fast scanning of a pulsed terahertz signal using an oscillating optical delay line. Review of Scientific Instruments, 78(2).

Jordens, C., \& Koch, M. (2008). Detection of foreign bodies in chocolate with pulsed terahertz spectroscopy. Optical Engineering, 47(3), 037003.

Kim, S. J., Born, B., Havenith, M., \& Gruebele, M. (2008). Real-time detection of protein-water dynamics upon protein folding by terahertz absorption spectroscopy. Angewandte ChemieInternational Edition, 47(34), 6486-6489.

Kim, G. J., Jeon, S. G., Kim, J. I., \& Han, S. T. (2010). A novel optical delay line using a rotating planar reflector for fast measurement of a terahertz pulse. Journal of the Korean Physical Society, 56(6), 1763-1766.

Kim, G. J., Jeon, S. G., Kim, J. I., \& Jin, Y. S. (2008). High speed scanning of terahertz pulse by a rotary optical delay line. Review of Scientific Instruments, 79(10).

Li, J. S. (2010). Optical parameters of vegetable oil studied by terahertz time-domain spectroscopy. Applied Spectroscopy, 64(2), 231-234.

Mantsch, H. H., \& Naumann, D. (2010). Terahertz spectroscopy: the renaissance of far infrared spectroscopy. Journal of Molecular Structure, 964(1-3), 1-4.

Markelz, A., Whitmire, S., Hillebrecht, J., \& Birge, R. (2002). THz time domain spectroscopy of biomolecular conformational modes. Physics in Medicine and Biology, 47(21), 3797-3805.

Maurer, L., \& Leuenberger, H. (2009). Terahertz pulsed imaging and near infrared imaging to monitor the coating process of pharmaceutical tablets. International Journal of Pharmaceutics, 370(1-2), 8-16.

McAuley, I., Young, L., Gradziel, M., Lanigan, W., O'Sullivan, C., Murphy, J.A., et al. (2006). Millimetre-wave and Terahertz Imaging Systems with Medical Applications. Infrared Millimeter Waves and 14th International Conference on Teraherz Electronics, 2006. IRMMW-THz 2006. Joint 31st International Conference on.

Morita, Y., Dobroiu, A., Kawase, K., \& Otani, C. (2005). Terahertz technique for detection of microleaks in the seal of flexible plastic packages. Optical Engineering, 44(1).

Nakajima, S., Hoshina, H., Yamashita, M., Otani, C., \& Miyoshi, N. (2007). Terahertz imaging diagnostics of cancer tissues with a chemometrics technique. Applied Physics Letters, 90(4).

Otsuka, M., Nishizawa, J., Shibata, J., \& Ito, M. (2010). Quantitative evaluation of Mefenamic Acid Polymorphs by terahertzChemometrics. Journal of Pharmaceutical Sciences, 99(9), 4048-4053.

Parasoglou, P., Parrott, E.P.J., Zeitler, J.A., Rasburn, J., Powell, H., Gladden, L.F., et al. (2009). Quantitative moisture content detection in food wafers. Infrared, Millimeter, and Terahertz Waves, 2009. IRMMW-THz 2009. 34th International Conference on.

Radhanpura, K., Hargreaves, S., Lewis, R. A., \& Henini, M. (2009). The role of optical rectification in the generation of terahertz radiation from GaBiAs. Applied Physics Letters, 94(25).

Redo-Sanchez, A., \& Zhang, X. C. (2011). Self-referenced method for terahertz wave time-domain spectroscopy. Optics Letters, 36(17), 3308-3310.

Redo-Sanchez, A., \& Zhang, X. C. (2011). Assessment of terahertz spectroscopy to detect antibiotic residues in food and feed matrices. Analyst, 136(8), 1733-1738.

Renk, K. F., Rogl, A., \& Stahl, B. I. (2007). Semiconductor-superlattice parametric oscillator for generation of sub-terahertz and terahertz waves. Journal of Luminescence, 125(1-2), 252-258.

Shen, Y. C. (2011). Terahertz pulsed spectroscopy and imaging for pharmaceutical applications: a review. International Journal of Pharmaceutics, 417(1-2), 48-60.

Tani, M., Watanabe, M., \& Sakai, K. (2002). Photoconductive twin dipole antennas for THz transceiver. Electronics Letters, 38(1), 5-6.

Wang, X. K., Cui, Y., Hu, D., Sun, W. F., Ye, J. S., \& Zhang, Y. (2009). Terahertz quasi-near-field real-time imaging. Optics Communications, 282(24), 4683-4687.

Wu, H., Heilweil, E. J., Hussain, A. S., \& Khan, M. A. (2007). Process analytical technology (PAT): effects of instrumental and compositional variables on terahertz spectral data quality to characterize pharmaceutical materials and tablets. International Journal of Pharmaceutics, 343(1-2), 148-158.

Yan, Z., Xinke, W., Ye, C., \& Wenfeng, S. (2009). THz quasi-near field focal plane imaging. Infrared, Millimeter, and Terahertz Waves, 2009. IRMMW-THz 2009. 34th International Conference on. 